\title{
A Homotopy Approach to the Feedback Stabilization of Linear Systems
}

\author{
Pierre T. Kabamba* \\ University of Michigan, Ann Arbor, Michigan \\ and \\ Richard W. Longman $\dagger$ and Sun Jian-Guo $\ddagger$ \\ Columbia University, New York, New York
}

\begin{abstract}
Constant-gain, fixed-order controllers for linear time-invariant systems are considered. A closed-form necessary and sufficient condition for the stabilizability of such a system by a controller of chosen order is established. This criterion is obtained by solving a constrained optimization problem and results in a system of nonlinear matrix equations. A method based on homotopy is proposed and studied to solve this system of nonlinear equations. The numerical implementation of the homotopy is discussed and various properties of the optimizing feedback control as a function of the homotopy parameter are established.
\end{abstract}

\section{Introduction}

$\mathbf{R}$ ICHARD Bellman often talked of "the curse of dimensionality." In the same spirit, we can identify "the curse of modern control theory." The most general and powerful modern control design methods, the linear-quadratic theory and the pole placement methods, require knowledge of the full state; in most cases, this implies using a full-order observer or filter in real-time operation-something that can easily invoke a curse of dimensionality on such designs. This is in stark contrast to classical design methods having low-dimensional controllers.

There is a need for effective fixed-order controller design methods to obtain controllers of prescribed intermediate dimension that can obtain some of the advantages of modern control theory without incurring the full-dimensionality "curse." One way to pose such problems is to consider the linear system with a quadratic cost problem, but to add the stipulation that the control must be obtained from the measurements via a dynamic feedback system whose dimension is prescribed in advance. Considerable attention has been given to this problem over the years and a few of the papers are cited here. ${ }^{1-10}$ Reference 1 presents a fairly comprehensive overview of the possible ways in which the quadratic cost, fixed-order controller problem can be posed. It also gives the first simple treatment of the finite-time versions of these problems. Extensive use of the design approach has been made by $\mathrm{Ly}^{2}$ employing his computer program SANDY. Hyland and Bernstein ${ }^{7-10}$ showed that the necessary conditions for optimality of a fixed-order controller are an elegant generalization of the well-known LQG design procedure obtained by introducing optimal oblique projections. Their results led to new computational procedures $^{8,10}$ and extensions to stochastic systems. ${ }^{11}$

Experience with fixed-order controller design has shown that often there is surprisingly little penalty paid for using controllers of dimension less than that of the system. Also, there are some indications that the lower-dimension con-

Received March 20, 1986; revision received Oct. 4, 1986. Copyright (C) American Institute of Aeronautics and Astronautics, Inc., 1987. All rights reserved.

*Assistant Professor of Aerospace Engineering.

$\dagger$ Professor of Mechanical Engineering.

$\ddagger$ Visiting Scholar (on leave from Nanjing Aeronautical Institue, Nanjing, China). trollers can be more robust than their full-order counterparts. However, the design of these controllers can be difficult, being plagued with problems of local minima and involving use of a gradient method that can have painfully slow convergence.

A still more fundamental difficulty occurs in designing controllers for unstable systems. To start the iterations of the gradient method, it is necessary to prescribe a starting value for the controller parameters that succeeds at stabilizing the system; otherwise, the gradient equations are invalid. Experience has shown that it can be very difficult to discover an initial stabilizing controller.

The primary purpose of this paper is to examine the fundamental question of existence of a stabilizing controller of specified order for a given system. This question is answered by the closed-form criterion of theorem 1 . This result is obtained by solving a constrained optimization problem. The criterion requires solving a system of nonlinear matrix equations. The secondary purpose of the paper is to study a homotopy method for solving this system of nonlinear matrix equations. The homotopy is performed on the parameter $\sigma$ when the system matrix $A$ is replaced by $A+\sigma I$. The numerical implementation of the homotopy is discussed and various properties of the optimizing feedback controller as a function of the homotopy parameter are established.

\section{Problem Formulation and Preliminary Results}

We consider a finite-dimensional linear time invariant system with minimal representation

$$
\begin{gathered}
\dot{x}_{s}=A_{s} x_{s}+B_{s} u \\
y=C_{s} x_{s}
\end{gathered}
$$

where $x_{s} \in \mid R^{n_{s}}$ is the system state vector, $u \in \mid R^{m_{s}}$ the input vector, $y \in \mid R^{p_{s}}$ the output vector, and $A_{s}, B_{s}, C_{s}$ real matrices of appropriate dimensions, $B_{s}$ and $C_{s}$ having full rank. The controller has the form

$$
\begin{gathered}
\dot{x}_{c}=A_{c} x_{c}+B_{c} y \\
u=C_{c} x_{c}+D_{c} y
\end{gathered}
$$

where $x_{c} \in \mid R^{n_{c}}$ is the controller state vector and $A_{c}, B_{c}, C_{c}$, $D_{c}$ real matrices of appropriate dimensions. The order $n_{c}$ of the controller ranges from 0 for direct output feedback to $n_{s}$ for full-order controller. 
The problem is then to determine whether matrices $A_{c}, B_{c}$, $C_{c}, D_{c}$ exist in Eq. (2) to make the closed-loop of Eqs. (1) and (2) asymptotically stable and, if they exist, to compute them.

Let $O_{n c}, I_{n c}$ denote the square zero and identity matrices of order $n_{c}$, respectively, and let $n=n_{s}+n_{c}, m=m_{s}+n_{c}$, and $p=p_{s}+n_{c}$. Define the matrices

$$
\begin{gathered}
A=\text { block diag }\left[A_{s}, O_{n c}\right] \in \mid R^{n \times n} \\
B=\text { block diag }\left[B_{s}, I_{n c}\right] \in \mid R^{n \times m} \\
C=\text { block diag }\left[C_{s}, I_{n c}\right] \in \mid R^{p \times n} \\
x=\left[x_{s}^{T}, x_{c}^{T}\right]^{T} \in \mid R^{n} \\
F=\left[\begin{array}{cc}
D_{c} & C_{c} \\
B_{c} & A_{c}
\end{array}\right] \in R^{m \times p}
\end{gathered}
$$

where, conveniently, all of the controller parameters have been gathered in matrix $F$. It is easily checked that the closed-loop equations are

$$
\dot{x}=(A+B F C) x
$$

and the problem can be reformulated as that of finding $F$ to make Eq. (4) asymptotically stable. This problem, together with the kindred pole assignment problem has received attention in the literature, but no closed-form solution has been given yet (see Refs. 12 and 13 and the references therein).

The questions of existence and computation of a stabilizing $F$ in Eq. (4) will be answered by solving a subsidiary reduced-order optimal control problem. The solution of the following problem will be useful.

Problem P. Find a controller of the form of Eqs. (2) and (3) for the system of Eq. (1) that minimizes

$$
\begin{aligned}
& J=\operatorname{tr}\left(F^{T} M F\right)+E\left\{\int_{0}^{\infty} x^{T} Q x e^{2 \sigma t} \mathrm{~d} t\right\} \\
& E[x(0)]=0 ; \quad E\left[x(0) x^{T}(0)\right]=X
\end{aligned}
$$

with respect to $F$; the symmetric matrices $X, Q$, and $M$ satisfy $X \geq 0, Q \geq 0$, and $M>0$.

In problem $\mathrm{P}$, the role of the parameter $\sigma$ is to ensure convergence of the integral in Eq. (5). Notice that even if Eq. (4) is unstable, choosing $\sigma$ negative and large enough will cause Eq. (5) to converge. To solve problem $P$ with $\sigma=0$, we will track its solution for $\sigma$ negative and let $\sigma$ tend to zero.

Solution $P$. (See Ref. 1 for a detailed development.) The necessary conditions for solving problem $\mathrm{P}$ are

$$
\begin{gathered}
A_{r} \triangleq A+B F C \\
\left(A_{r}+\sigma I\right)^{T} K+K\left(A_{r}+\sigma I\right)+Q=0 \\
\left(A_{r}+\sigma I\right) \Lambda+\Lambda\left(A_{r}+\sigma I\right)^{T}+X=0 \\
\frac{1}{2} \frac{\partial J}{\partial \dot{F}}=M F+B^{T} K \Lambda C^{T}=0
\end{gathered}
$$

where the square symmetric matrices $K$ and $\Lambda$ satisfy $K \geq 0$ and $\Lambda \geq 0$.

The usual gradient method for solving problem $\mathrm{P}$ consists of starting with a-stabilizing $F$, obtaining $A_{r}$ from Eq. (6), solving Eqs. (7) and (8) for $K$ and $\Lambda$, and using the result to find the cost gradient $\partial J / \partial F$ in Eq. (9); then let $F$ be incremented to decrease $J$ along the gradient by adding $-c \partial J / \partial F$, where $c>0$ is adjusted to maintain convergence at each step.
It is important to notice that in the context of this paper, the solution of problem $\mathrm{P}$ does not constitute a control design method per se. Problem $P$ is only a tool to help us answer the questions of existence and computation of a stabilizing $F$ in Eq. (4).

\section{Stabilizability}

If the dimension of the controller [Eq. (2)] for system of Eq. (1) is too low, it is quite possible that there exists no controller that can stabilize the system. This section is devoted to establishing a closed-form necessary and sufficient condition for the stabilizability of the system of Eq. (1) by a controller having the form of Eq. (2) with given dimension $n_{c}$.

There is a basic theorem of Liapunoy stating that a matrix $A$ corresponds to an asymptotically stable differential equation if and only if there exists a $K>0$ satisfying $A^{T} K+K A+I=0$ (or, if we wish, a $\Lambda>0$ satisfying $A \Lambda+\Lambda A^{T}+I=0$ ). We can show the following stability result reminiscent of the Liapunov result.

Theorem 1 . The triple $\left(A_{s}, B_{s}, C_{s}\right)$ is stabilizable by a finite linear feedback controller $F$ of the form of Eqs. (2) and (3) with dimension $n_{c}$ if and only if

$$
\begin{gathered}
\overline{\mathscr{F}}(K, \Lambda) \triangleq A^{T} K+K A-\bar{C} \Lambda K \bar{B} K-K \bar{B} K \Lambda \bar{C}+I=0 \\
\overline{\mathcal{G}}(K, \Lambda) \triangleq A \Lambda+\Lambda A^{T}-\bar{B} K \Lambda \bar{C} \Lambda-\Lambda \bar{C} \Lambda K \bar{B}+I=0
\end{gathered}
$$

admit a solution with $K>0$ and $\Lambda>0$. In this case,

$$
F=-\bar{B}^{T} K \Lambda \bar{C}^{T}
$$

is a stabilizing solution. Matrix $\bar{C}=C^{T} C, \bar{B}=B B^{T}, I$ is the $n_{s}+n_{c}$ identity matrix and $A, B, C$ are given by Eq. (3).

Remark. Note that, if there is no control, we can set $n_{c}=0$ and $B=0$ and the above theorem then reduces to the cited Liapunov result. As in the Liapunov result, the identity matrices in Eqs. (10) can be replaced by any positive definite matrices (not necessarily the same). Note also that the Riccati equations are a generalization of the Liapunov equations by including a quadratic term and that Eqs. (10) similarly generalize the Liapunov equations by including cubic terms.

Proof. If the equations have a solution $K>0$, then substitution of the given $F$ into the $K$ equation produces

$$
(A+B F C)^{T} K+K(A+B F C)+I=0
$$

From the Liapunov result, this implies that Eq. (4) is asymptotically stable.

Conversely, if $\left(A_{s}, B_{s}, C_{s}\right)$ is stabilizable, then there exists an $F$ such that Eq. (4) is asymptotically stable. Choosing this $F$ will give the following cost a finite value:

$$
J=\operatorname{tr}\left(F^{T} F\right)+E \int_{0}^{\infty} x^{T} x \mathrm{~d} t
$$

when the initial conditions of Eq. (4) are specified as $E\left[x_{0}\right]=0$ and $E\left[x_{0} x_{0}^{T}\right]=I$. In fact,

$$
\begin{gathered}
J=\operatorname{tr}\left(F^{T} F\right)+E\left(x_{0}^{T} \Phi x_{0}\right)=\operatorname{tr}\left(F^{T} F\right)+\operatorname{tr}(\Phi) \\
\Phi=\int_{0}^{\infty} \exp \left(A^{T} t\right) \quad \exp (A t) \mathrm{d} t
\end{gathered}
$$

where the integral $\Phi$ exists since $A$ is asymptotically stable. In addition, $J$ diverges if $F$ is not stabilizing.

The set of stabilizing controllers of order $n_{c}, F_{n c}$ is an open set; thus, by the above, $J$ is finite for all such controllers and $J \rightarrow \infty$ as $F$ approaches the boundary of the closure of $\boldsymbol{F}_{n c}$. Within $F_{n c}, J$ is a smooth function of $F$. To see this, note 
that the $\Phi$ in $J$ satisfies

$$
(A+B F C)^{T} \Phi+\Phi(A+B F C)+I=0
$$

or

$$
\left[(A+B F C)^{T} \otimes I+I \otimes(A+B F C)\right] \quad \operatorname{col}(\Phi)=-\operatorname{col}(I)
$$

where $\operatorname{col}(\Phi)$ is a column matrix of the rows of $\Phi$ entered row by row and $\otimes$ denotes the Kronecker product. ${ }^{16}$ The coefficient matrix is nonsingular since $F$ is stabilizing and its eigenvalues are $\lambda_{i}+\lambda_{j}$ for all $i, j$ where the $\lambda$ are eigenvalues of $A+B F C$. Therefore, a minimum of $J$ exists for some $F \in F_{n c}$. This minimum must satisfy the necessary conditions of Eqs. (7-9) with $\sigma=0, Q=I, X=I$, and $M=I$. Solving Eq. (9) for $F$ gives the $F$ of the theorem and substituting Eq. (9) into Eqs. (7) and (8) produces Eqs. (10).

Theorem 1 is obtained by solving an optimization problem. It is well known that the minimum of a differentiable convex function on a convex set is unique. As a point of interest, note that the set of stabilizing controllers is not convex. Let $n=m=p=2, A_{1}=A+B F_{1} C, A_{2}=A+B F_{2} C$, and consider $A_{\alpha}=A+B F C$ for $F=\alpha F_{1}+(1-\alpha) F_{2}, \quad 0 \leq \alpha \leq 1$. Consider $A=0, B=I, C=I$, and

$$
F_{1}=\left[\begin{array}{cc}
0 & -1 \\
1 & -1
\end{array}\right] \quad F_{2}=\left[\begin{array}{cc}
-1 & +2+\sqrt{3} \\
-2+\sqrt{3} & 0
\end{array}\right]
$$

making $A_{1}$ and $A_{2}$ stable, since their determinants are positive and their traces negative. If $\alpha$ is set to $1 / 2, A_{\alpha}$ produces a matrix whose determinant is $-1 / 4$, which indicates instability.

\section{Numerical Methods for Obtaining Stabilizing Controllers}

There are various possible approaches to obtaining a stabilizing controller of a prescribed dimension. One approach is to use pole placement methods for fixed-order controllers as discussed in Refs. 7 and 8 . Another approach is to use finite-time Liapunov equations in place of Eqs. (7) and $(8)$, since solutions then exist even in the unstable case and to let the time tend to infinity during the solution process. ${ }^{2}$ In this paper, we propose to compute positive definite solutions of Eqs. (10) and obtain a stabilizing controller of Eq. (11). We have tried several computational methods.

\section{Newton's Iteration}

One possible way of solving Eqs. (10) is to use Newton's iteration. If the $(K, \Lambda)$ of Eqs. (10) are perturbed to $(K+\delta K, \Lambda+\delta \Lambda)$, the linearized equations in $(\delta K, \delta \Lambda)$ are

$$
\begin{aligned}
& (A-\bar{B} K \Lambda \bar{C})^{T} \delta K+\delta K(A-\bar{B} K \Lambda \bar{C}) \\
& -(\Lambda \bar{C})^{T} \delta K(K \bar{B})^{T}-(K \bar{B}) \delta K(\Lambda \bar{C}) \\
& -\bar{C} \delta \Lambda(K \bar{B} K)-(K \bar{B} K) \delta(\Lambda \bar{C})=-\bar{F}(K, \Lambda)
\end{aligned}
$$

and

$$
\begin{aligned}
& (A-\bar{B} K \dot{\Lambda} \bar{C}) \delta \Lambda+\delta \Lambda(A-\bar{B} K \Lambda \bar{C})^{T} \\
& -(K \bar{B})^{T} \delta \dot{\Lambda}(\Lambda \bar{C})^{T}-(\Lambda \bar{C}) \delta \Lambda(K \bar{B}) \\
& -\bar{B} \delta K(\Lambda \bar{C} \Lambda)-(\dot{\Lambda} \bar{C} \Lambda) \delta K \bar{B}=-\overline{\mathcal{G}}(K, \Lambda)
\end{aligned}
$$

The procedure consists of picking $K$ and $\Lambda$ to be positive definite, solving Eq. (15) for $\delta K$ and $\delta \Lambda$, incrementing $(K, \Lambda)$ by $(\delta K, \delta \Lambda)$, and iterating until convergence: Unfortunately, this method requires good initial guesses of $K_{0}, \Lambda_{0}$ and at each step $K$ and $\Lambda$ are not guaranteed to remain positive definite.

\section{Differential Equation}

There is another possible method for solving Eqs. (10). One way to solve the algebraic Riccati equation is to integrate the Riccati differential equation from any positive definite initial condition until the solution reaches steady state. One wonders whether the same approach would work for solving our coupled third-degree "Riccati" equations.

$$
\begin{gathered}
\dot{K}=A^{T} K+K A-\bar{C} \Lambda K \bar{B} K-K \bar{B} K \Lambda \bar{C}+I \\
\dot{\Lambda}=A \Lambda+\Lambda A^{T}-\bar{B} K \Lambda \bar{C} \Lambda-\Lambda \bar{C} \Lambda K \bar{B}+I
\end{gathered}
$$

Starting from zero initial conditions, numerical experiment with this algorithm indicates that sometimes it converges and other times does not. If the method is to succeed, there must be an asymptotically stable positive definite equilibrium solution to Eqs. (16) and the initial conditions must be within the domain of attraction. We can establish two properties of the method.

First, if $K\left(t_{0}\right) \geq 0$ and $\Lambda\left(t_{0}\right) \geq 0$ for some $t_{0}$, then $K(t) \geq 0$ and $\Lambda(t) \geq 0$ for all $t \geq t_{0}$, so that solutions can never escape from the positive semidefinite domain. If $K$ (or $\Lambda$ ) were to leave the set of positive definite matrices, at the point of departure, one of its eigenvalues would be zero; call it $\nu$ and let $x$ be the associated eigenvector of unit length. Taking the product with $x^{T}$ from the left and $x$ on the right of each side of the $K$ equation in Eq. (16) produces $x^{T} \dot{K} x=x^{T} x=1$, using $K x=0$. But $x^{T} \dot{K} x=\dot{\nu}$ so that $\dot{\nu}=1$. Therefore, if $K$ ever reaches the boundary of the positive definite region, it cannot cross the boundary. The result for $\Lambda$ is similar.

In order to gain some insight into the stability or lack of stability of the positive definite equilibrium solutions to Eqs. (16), consider the following. Replace the unit matrices in Eqs. (16) by $\epsilon I$, where $\epsilon>0$ is a small parameter [recall that in Eqs. (10), as in Liapunov's criterion, the identity matrices can be replaced by any positive definite matrices, no matter how small]. When $\epsilon=0$, the equilibrium conditions are satisfied by $K=\Lambda=0$ and, therefore, we expect a nearby solution when $\epsilon$ is a small positive number. For sufficiently small $\epsilon$, this solution should be obtainable from

$$
\begin{aligned}
& A^{T} K+K A+\epsilon I=0 \\
& A \Lambda+\Lambda A^{T}+\epsilon I=0
\end{aligned}
$$

To establish the stability of an equilibrium solution of a nonlinear equation, one can linearize about the equilibrium. If the linearized equations are asymptotically stable (unstable), then the equilibrium solution of the nonlinear equation is asymptotically stable (unstable). The linearized equations have the form

$$
\left[\begin{array}{c}
\dot{\delta} k \\
\dot{\delta} \lambda
\end{array}\right]=\alpha(k, \lambda)\left[\begin{array}{l}
\delta k \\
\delta \lambda
\end{array}\right]
$$

where $k$ and $\lambda$ are vectors of order $n^{2}$ containing the elements of $K$ and $\Lambda ; \delta k$ and $\delta \lambda$ similarly contain the perturbation of $K$ and $\Lambda$. Recognizing that $K$ and $\Lambda$ are of order $\epsilon$, matrix $Q$ can be written in the form

$$
Q(k, \lambda)=\left[\begin{array}{cc}
A^{T} \otimes I+I \otimes A & 0 \\
0 & A \otimes I+I \otimes A^{T}
\end{array}\right]+\epsilon \Delta Q
$$

and for sufficiently small $\epsilon$, the eigenvalues of $Q$ will be arbitrarily close to those of the first term on the right-hand side. But the eigenvalues of this are just those of Eqs. (17), which are $\mu_{i}+\mu_{j}$ for all $i, j$ where the $\mu_{k}$ are eigenvalues of $A$. Therefore, an unstable system matrix $A$ can produce an unstable equilibrium solution to Eqs. (16) for sufficiently small $\epsilon$. 


\section{The $\sigma$-Homotopy}

As a third method for solving Eqs. (10), we tried a homotopy method. Homotopy methods have been increasingly used in control ${ }^{14}$ and are based on the following idea. (See Refs. 14 and 15 for details.) Consider the differentiable function $F: R^{n} \rightarrow R^{n}: \xi \rightarrow F(\xi)$ and suppose we want to solve the system of nonlinear equations $F(\xi)=0$. Assume we are able to find a differentiable function $G: R^{n} \times\left[\sigma_{0}, \sigma_{1}\right]$ : $(\xi, \sigma) \rightarrow G(\xi, \sigma)$ such that $G\left(\xi, \sigma_{1}\right) \equiv F(\xi)$ and the equation $G\left(\xi, \sigma_{0}\right)=0$ has a known solution $\xi_{0}$. The idea is then to track the solution of the equation

$$
G(\xi, \sigma)=0
$$

as a function of $\sigma \in\left[\sigma_{0}, \sigma_{1}\right]$, starting at $\xi=\xi_{0}$ for $\sigma=\sigma_{0}$ and ending at $\xi^{*}$, the solution of our problem for $\sigma=\sigma_{1}$. One way to track the solution of Eq. (18) is to solve the differential equation with the initial conditions

$$
\begin{gathered}
\left(\frac{\partial G}{\partial \xi}\right)^{T}\left(\frac{\mathrm{d} \xi}{\mathrm{d} \sigma}\right)+\frac{\partial G}{\partial \sigma}=0 \\
\xi\left(\sigma_{0}\right)=\xi_{0}
\end{gathered}
$$

which is obtained by differentiating-Eq. (18) with respect to a. Equation (19) has solutions satisfying $G(\xi, \sigma)=$ const, indicating that if during the course of integrating Eq. (19) numerical errors cause $G(\xi, \sigma) \neq 0$, these errors will not be eliminated. As a consequence, because of numerical errors, a better way to track the solution of Eq. (18) is to integrate

$$
\begin{gathered}
\left(\frac{\partial G}{\partial \xi}\right)^{T}\left(\frac{\mathrm{d} \xi}{\mathrm{d} \sigma}\right)+\left(\frac{\partial G}{\partial \sigma}\right)+\beta G=0 \\
\xi\left(\sigma_{0}\right)=\xi_{0} \\
\beta>0
\end{gathered}
$$

which has a solution satisfying $G(\xi, \sigma)=G\left(\xi_{0}, \sigma_{0}\right) e^{-\beta\left(\sigma-\sigma_{0}\right)}$, indicating that if in the course of integration numerical errors cause $G(\xi, \sigma) \neq 0$, the term $\beta G$ in Eqs. (20) will tend to eliminate these errors. This stabilization procedure is similar to one used by Baumgarte to simulate dynamical systems with constraints. ${ }^{17}$

In the context of Eqs. (10), we used a homotopy on the parameter $\sigma$ when $A$ is replaced by $A+\sigma I$. Choose $\sigma_{0}$ such that $A+\sigma_{0} I$ is asymptotically stable. Problem $\mathrm{P}$ can then be solved using the first-order gradient method with $Q=X=I$, resulting in $K_{0}, \Lambda_{0}$. The solution of the equations

$$
\begin{aligned}
& \mathcal{F}(K, \Lambda, \sigma) \triangleq(A+\sigma I)^{T} K+K(A+\sigma I)-\bar{C} \Lambda K \bar{B} K-K \bar{B} K \Lambda \bar{C}+I=0 \\
& \mathcal{G}(K, \Lambda, \sigma) \triangleq(A+\sigma I) \Lambda+\Lambda(A+\sigma I)^{T}-\bar{B} K \Lambda \bar{C} \Lambda-\Lambda \bar{C} \Lambda K \bar{B}+I=0
\end{aligned}
$$

are then tracked as functions of $\sigma$ starting at $(K, \Lambda)=$ $\left(K_{0}, \Lambda_{0}\right)$ for $\sigma=\sigma_{0}$ and ending at the solution of Eqs. (10) for $\sigma=0$.

In the following, we will develop numerical approaches to handling the homotopy, develop some theoretical properties of the homotopy, and evaluate its behavior and its potential for tracking difficulties by means of studying simple examples in detail.

\section{Numerical Implementation of the $\sigma$ Homotopy}

There are various ways of tracking the solutions of Eqs. (21) as functions of $\sigma$. The simplest way from a programming standpoint is to increment $\sigma$ by a small step, use the $K$ and $\Lambda$ as a.starting value at the new $\sigma$, and apply a gradient or Newton method, the latter of which might be preferable because of its convergence properties in the neighborhood of a solution.

However, a more sophisticated approach is to develop and use a differential equation similar to Eq. (20) that tracks the solution from $\sigma=\sigma_{0}$ to $\sigma=0$,

$$
\begin{aligned}
& (A+\sigma \bar{I}-\bar{B} K \Lambda \bar{C})^{T} \dot{K}+\stackrel{\circ}{K}(A+\sigma I-\bar{B} K \Lambda \bar{C}) \\
& -(\Lambda \bar{C})^{T} \dot{K}(K \bar{B})^{T}-(K \bar{B}) \stackrel{K}{K}(\Lambda \bar{C})-\bar{C} \AA(K \bar{B} K)
\end{aligned}
$$

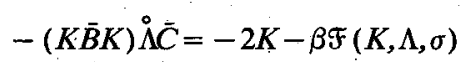

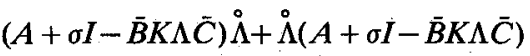

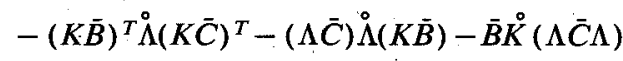

$$
\begin{aligned}
& -(\Lambda \bar{C} \Lambda) \stackrel{K}{K} \bar{B}=-2 \Lambda-\beta \mathcal{G}(K, \Lambda, \sigma)
\end{aligned}
$$

where $\stackrel{\circ}{K}=\mathrm{d} K / \mathrm{d} \sigma ; \stackrel{\circ}{\Lambda}=\mathrm{d} \Lambda / \mathrm{d} \sigma, F(K, \Lambda, \sigma)$ and $\mathcal{S}(K, \Lambda, \sigma)$ are defined in Eqs. (21), and $\beta$ is a convergence factor that can be adjusted for stability of tracking.

This $\sigma$-homotopy tracking procedure has the desirable properties that-sophisticated numerical integration packages with self-adjusting step sizes can be used for accurate tracking and they have the stability factor $\beta$ to correct accumulated numerical errors. Note that there are some differential equation solving routines that do not require isolation of $K$ and $\AA$ and hence might be applied directly to Eq. (22).

In the event that one wishes to start at $\sigma_{0}=-\infty$ from the known unique solutions $K=0$ and $\Lambda=0$ (obtained below), one can change variables from $\sigma$ to $t=-(\sigma-1)^{-1}$ and solve the differential equations from $t=0$ to 1 . Starting with $t \mathfrak{F}(K, \Lambda,(t-1 / t)=0$ and $t \mathcal{S}(K, \lambda,(\epsilon-1 / t)=0$, differentiating with respect to $t$, and introducing a stabilizing factor $\beta$ as before produces

$$
\begin{aligned}
& 2(\dot{t}-1) \dot{K}+t\left[(A-\bar{B} K \Lambda \bar{C})^{T} \dot{K}+\dot{K}(A-\bar{B} K \Lambda \bar{C})\right. \\
& -(\Lambda \bar{C})^{T} \dot{K}(K \bar{B})^{T}-(K \bar{B}) \dot{K}(\Lambda \bar{C})-\bar{C} \dot{\Lambda}(K \bar{B} K) \\
& -(K \bar{B} K) \dot{\Lambda} \bar{C}]=-(1+t \beta)\left[(A-\bar{B} K \Lambda \bar{C})^{T} K\right. \\
& +K(A-\bar{B} K \Lambda \bar{C})+I+2 K]+2 \beta K
\end{aligned}
$$

$$
\begin{gathered}
2(t-1) \dot{\Lambda}+t\left[(A-\bar{B} K \Lambda \bar{C}) \dot{\Lambda}+\dot{\Lambda}(A-\bar{B} K \Lambda \bar{C})^{T}\right. \\
-(K \bar{B})^{T} \dot{\Lambda}(\Lambda \bar{C})^{T}-(\Lambda \bar{C}) \dot{\Lambda}(K \bar{B})-\bar{B} \dot{K}(\Lambda \bar{C} \Lambda) \\
-(\Lambda \bar{C} \Lambda) \dot{K} \bar{B}]=-(1+t \beta)[(A-\bar{B} K \Lambda \bar{C}) \Lambda \\
\left.+\Lambda(A-\bar{B} K \Lambda \bar{C})^{T}+I+2 \Lambda\right]+2 \beta \Lambda
\end{gathered}
$$

where $\dot{K}=\mathrm{d} K / \mathrm{d} t$ and $\dot{\Lambda}=\mathrm{d} \Lambda / \mathrm{d} t$.

\section{The Optimal Cost along the $\sigma$ Homotopy}

As the first of several properties of the $\sigma$ homotopy to be established, let us consider the optimal cost as a function of $\sigma$. The cost for any stabilizing $F$ and $\sigma$ for problem $\mathrm{P}$ with $M=I$ and $X=I$ is given by

$$
\begin{array}{r}
J(F, \sigma)=\operatorname{tr}\left(F^{T} F\right)+\operatorname{tr}(K) \\
\Gamma(F, K, \sigma) \triangleq(A+\sigma I+B F C)^{T} K(F, \sigma) \\
+K(F, \sigma)(A+\sigma I+B C)+Q=0
\end{array}
$$


Then, $\mathrm{d} J=(\partial J / \partial \sigma) \mathrm{d} \sigma+\operatorname{tr}\left[(\partial J / \partial F)^{T} \mathrm{~d} F\right]$. For the optimal $F$ at any $\sigma, \partial J / \partial F=0$, which implies

$$
\frac{\mathrm{d} J^{*}}{\mathrm{~d} \sigma}=\frac{\partial J^{*}}{\partial \sigma}
$$

where the asterisk on $J$ signifies optimality and the partial derivative signifies a derivative with respect to explicit dependence on $\sigma$, i.e., through $K$, not through $F$. Appending the constraint $\Gamma=0$ to the optimal cost using Lagrange multipliers $\Lambda, J^{\prime}(F, \sigma)=\operatorname{tr}\left(F^{T} F\right)+\operatorname{tr} K+\operatorname{tr}(\Lambda \Gamma)$ and differentiating produces $\partial J^{\prime} / \partial \sigma=\operatorname{tr}(\Lambda K+K \Lambda)$ or

$$
\mathrm{d} J^{*} / \mathrm{d} \sigma=2 \operatorname{tr}(\Lambda K)
$$

where $\Lambda$ and $K$ are optimal for each $\sigma$.

We will show that this expression implies that the optimal cost is a monotonically nondecreasing function of $\sigma$ and that, if $Q>0$ or $X>0$, the optimal cost is monotonically increasing. To do so we need the following properties for symmetric matrices $A$ and $B: 1)$ if $A>0$ and $B \geq 0$ with $B \neq 0$ or if $A \geq 0$ and $B>0$ with $A \neq 0$, then $\operatorname{tr}(A B)>0$; and 2) if $A \geq 0$ and $B \geq 0$, then $\operatorname{tr}(A B) \geq 0$. To prove this, note that the characteristic equation of a matrix, and hence its trace which gives the second coefficient, is invariant under a similarity transformation. Let $\Pi$ be a unitary matrix that diagonalizes $A, \Pi^{T} A \Pi=\mu=\operatorname{diag}\left(\mu_{1}, \ldots, \mu_{n}\right)$, and $\Pi^{T} B \Pi=\bar{B}=\left[\bar{b}_{i j}\right]$. Then,

$$
\operatorname{tr}(A B)=\operatorname{tr}\left(\Pi^{T} A B \Pi\right)=\operatorname{tr}(\mu \bar{B})=\sum_{i} \mu_{i} \bar{b}_{i i}
$$

If $B>0$, then $x^{T} B x>0$ for all $x \neq 0$ and $y^{T} \Pi^{T} B \Pi y=y^{T} \bar{B} y>0$ for all $y \equiv 0$, and choosing $y$ as columns of the identity matrix establishes that $\bar{b}_{i i}>0$. This makes $\operatorname{tr}(A B)>0$ if $A>0, B>0$, since this implies $\mu_{i}>0$ and $\bar{b}_{i i}>0$. If $A \geq 0$ and $B>0$ with $A \neq 0$, then all $\mu_{i} \geq 0$ and $\bar{b}_{i i}>0$ with some $\mu_{i} \neq 0$,

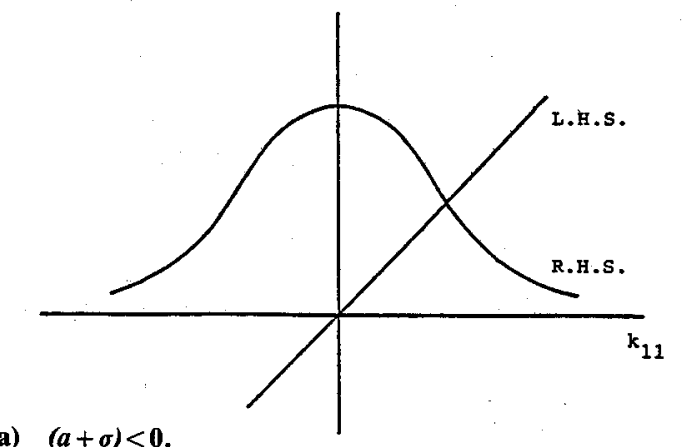

a) $(a+\sigma)<0$.

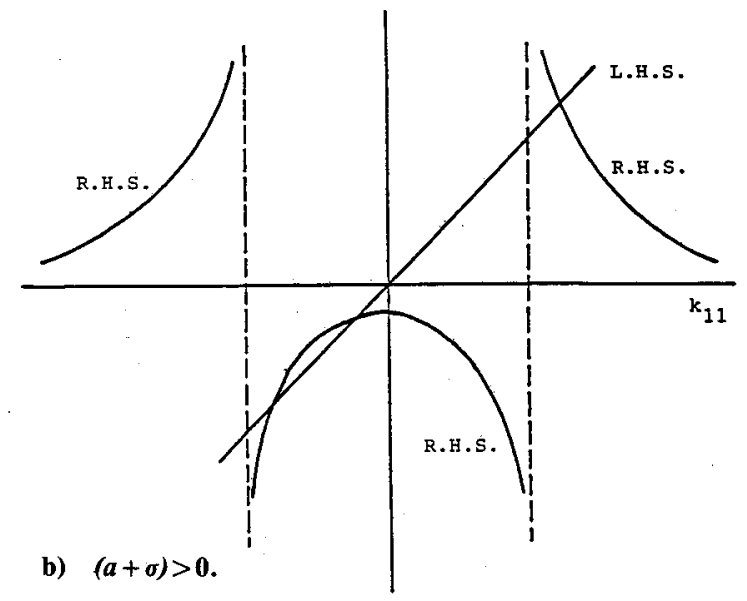

Fig. 1 Solutions to Eq. (32) by finding the intersections of the graphs of the right- and left-hand sides (R.H.S. and L.H.S). which implies $\operatorname{tr}(A B)>0$. Since $\operatorname{tr}(A B)=\operatorname{tr}\left(B^{T} A^{T}\right)$, the same proof can be used to establish the first half of property 1 .

Theorem 2. If $A_{r}+\sigma I$ of Eq. (6) is asymptotically stable, the optimal cost $J^{*}$ for problem $\mathrm{P}$ has the following monotonicity properties as a function of $\sigma$ :

1) $\mathrm{d} J^{*} / \mathrm{d} \sigma=2 \operatorname{tr}(\Lambda K) \geq 0$.

2) If $Q>0$ or if $X>0$, then $\mathrm{d} J^{*} / \mathrm{d} \sigma=2 \operatorname{tr}(\Lambda K)>0$.

Proof. Since $A_{r}+\sigma I$ is asymptotically stable, by Liapunov's theorem positive semidefiniteness (positive definiteness) of $Q$ or $X$ implies positive semidefiniteness (positive definiteness) of $K$ or $\Lambda$, respectively.

Properties of the Optimal Dynamic Controllers as a Function of $\sigma$

Various characteristics of the optimal controller (for problem $\mathrm{P}$ with $M=I$ ) as a function of $\sigma$ can be developed analytically when the controller is a compensator, i.e., $n_{c}>0$. In this case, $Q$ and $X$ have the form $Q=$ block $\operatorname{diag}\left[Q_{s}, O_{n c}\right], X=$ block diag $\left[X_{s}, O_{n c}\right]$ where $Q_{s}$ and $X_{s}$ correspond to $x_{s}$. The optimal $K$ and $\Lambda$ satisfy Eqs. (6-9). By making a change of variables to $t=-1 / \sigma$, we can study the behavior of the solutions as $\sigma \rightarrow-\infty$ by letting $t \downarrow 0^{+}$. The equations become

$$
\begin{aligned}
& t\left[(A-\bar{B} K \Lambda \bar{C})^{T} K+K(A-\bar{B} K \Lambda \bar{C})+Q\right]-2 K=0 \\
& t\left[(A-\bar{B} K \Lambda \bar{C}) \Lambda+\Lambda(A-\bar{B} K \Lambda \bar{C})^{T}+X\right]-2 \Lambda=0
\end{aligned}
$$

At $t=0$, we conclude that $K=\Lambda=0$. Such a result might be intuitively reasonable because the system transients die away with an infinitesimal time constant and there is thus no need for the feedback control to exert effort to get the state to zero.

To study the solution in the neighborhood of $t=0$, differentiate Eq. (25) to obtain the following differential equations:

$$
\begin{aligned}
& -2 \dot{K}+(A-\bar{B} K \Lambda \bar{C})^{T}(K+t \dot{K})+(K+t \dot{K})(A-\bar{B} K \Lambda \bar{C})+Q \\
& -t\left[\bar{C}(\dot{K} \Lambda+K \dot{\Lambda})^{T} \bar{B} K+K \bar{B}(K \dot{\Lambda}+K \dot{\Lambda}) \bar{C}\right]=0 \\
& -2 \dot{\Lambda}+(A-\bar{B} K \Lambda \bar{C})(\Lambda+t \dot{\Lambda})+(\dot{\Lambda}+t \Lambda)(A-\bar{B} K \Lambda \bar{C})^{T}+X \\
& -t\left[\bar{B}(\dot{K} \Lambda+K \dot{\Lambda}) \bar{C} \Lambda+\Lambda \bar{C}(\dot{K} \Lambda+K \dot{\Lambda})^{T} \bar{B}\right]=0
\end{aligned}
$$

[Note that these same differential equations in $t$ should be obtainable from the differential equations in $\sigma$ presented in Eqs. (22) (with $\mathcal{F}$ and $\mathcal{S}$ estimated from the right-hand side since they are zero along the optimal solution) by substituting $\sigma=-1 / t$, but that in order to obtain the behavior near zero by this approach one must eliminate various terms using $F=0$ and $\mathcal{G}=0$.] Setting $t=0, K(0)=\Lambda(0)=0$ in Eqs. (26) gives

$$
\dot{K}(0)=1 / 2 Q, \quad \dot{\Lambda}(0)=1 / 2 X
$$

Then, for small $t, K(t) \approx K(0)+\dot{K}(0) t=1 / 2 Q t$ and $\Lambda(t) \approx$ $1 / 2 X t$, in which case $K(t) \approx(t / 2) \operatorname{diag}\left(Q_{s}, O_{n c}\right)$ and $\Lambda(t) \approx(t / 2) \operatorname{diag}\left(X_{s}, O_{n c}\right)$. Substituting such a form into Eq. (9) for the controller parameters $F$ establishes that the partitions $C_{c}$ and $A_{c}$ are zero [see Eq. (2)], which means that the controller is a direct output feedback one.

We conclude that when $\sigma$ is sufficiently negative, there is a unique solution to the necessary conditions [Eqs. (6-9)] and that this solution is of the form of a direct output feedback controller. No fixed-order controllers of prescribed dimension $n_{c}>0$ are optimal for such $\sigma$-the mathematics can generate controller dynamics of this dimension, but the controller state is unobservable in the control signal and, therefore, the optimal fixed-order controller generates the same control signal as the direct output feedback one. 

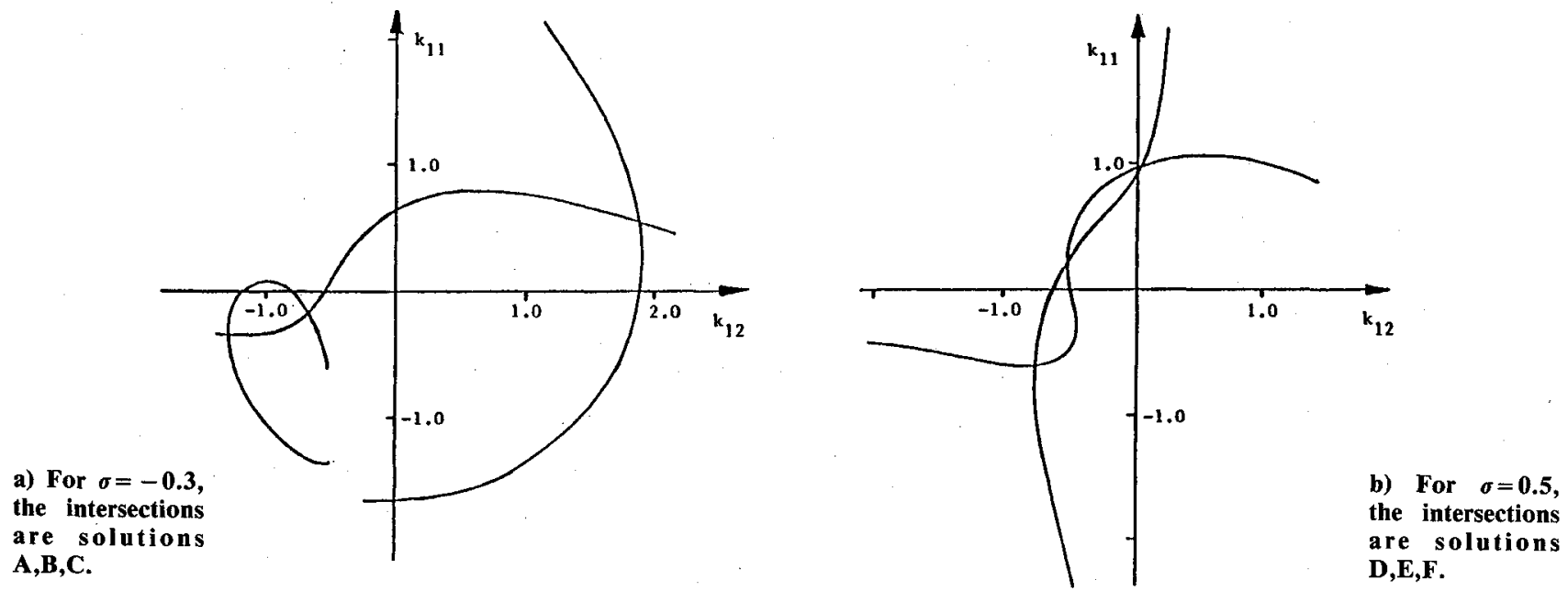

Fig. 2 Check to determine the number of possible solutions.

Equations (7) and (8) define $K$ and $\Lambda$ as implicit functions of $\sigma$. The left-hand sides of these equations are polynomials in the unknown components and are infinitely continuously differentiable. Therefore, $K$ and $\Lambda$ can be expressed as functions of $\sigma$ that are again infinitely continuously differentiable in the neighborhood of every point where the gradient of the left-hand side of Eqs. (7) and (8) with respect to $K$ and $\Lambda$ is nonsingular.

As a result, the differential equation (26) that tracks the values of $K$ and $\Lambda$ as functions of $\sigma$, starting from the unique initial value of interest when $\sigma \rightarrow-\infty$, will satisfy a uniqueness theorem for this solution on any interval in $\sigma$ until a value $\sigma$ is reached where Eqs. (26) cannot be uniquely solved for $\dot{K}$ and $\dot{\Lambda}$. At such a $\vec{\sigma}$, it is possible to have a bifurcation of the solution that emanated from the given initial conditions.

Let us examine the nature of the solution between $-\infty$ and $\tilde{\sigma}$. Assume that $M=\operatorname{diag}\left(M_{1}, M_{2}\right)$, where $M_{1}$ is $n_{s} \times n_{s}$ and $M_{2}$ is $n_{c} \times n_{c}$, and examine problem $\mathrm{P}$ using the differential equations (26). Let $K$ and $\Lambda$ be partitioned as

$$
K=\left[\begin{array}{ll}
K_{1} & K_{3} \\
K_{3}^{T} & K_{2}
\end{array}\right], \quad \Lambda=\left[\begin{array}{ll}
\Lambda_{1} & \Lambda_{3} \\
\Lambda_{3}^{T} & \Lambda_{2}
\end{array}\right]
$$

We will demonstrate that if $K$ and $\Lambda$ have the form $K=\operatorname{diag}\left(K_{1}, 0\right)$ and $\Lambda=\operatorname{diag}\left(\Lambda_{1}, 0\right)$ for some $\sigma\left(K_{1}\right.$ and $\Lambda_{1}$ are $\left.n_{c} \times n_{c}\right)$ and we know this holds as $\sigma \rightarrow-\infty$, then the form will be maintained for all larger $\sigma$ until such a point as $\bar{\sigma}$ occurs. In other words, the unique solution starting at $\sigma \rightarrow-\infty$ remains a direct output feedback solution until $\sigma$ reaches $\bar{\sigma}$, at which point a bifurcation of solutions may occur. The new solution can introduce a controller of dimension $n_{c}>0$, while the original branch can maintain the direct output feedback structure. Of course, as the new solution is tracked as a function of $\sigma$, a new point such as $\bar{\sigma}$ may be encountered, allowing another increase in the dimension of the optimal controller.

To demonstrate that the forms of $K$ and $\Lambda$ are maintained until reaching $\bar{\sigma}$, substitute such forms into Eqs. (26) and examine the four partitions of the resulting equations. The first partition produces equations of exactly the same form as Eqs. (26), except that all of the matrices have been replaced by their upper left-hand partitions. The other equations yield

$$
\begin{array}{ll}
\dot{K}_{2}=0 & \alpha_{11} \dot{K}_{3}+\alpha_{12} \dot{\Lambda}_{3}=0 \\
\dot{\Lambda}_{2}=0 & \alpha_{21} \dot{K}_{3} \beta_{2}+\alpha_{22} \dot{\Lambda}_{3}=0
\end{array}
$$

where

$$
\begin{aligned}
& \alpha_{11}=t\left(A-B M_{1}^{-1} B^{T} K_{1} \Lambda_{1} C^{T} C\right)^{T}-2 I \\
& \alpha_{22}=\alpha_{11}^{T} \\
& \alpha_{12}=-t K_{1} B M_{1}^{-1} B^{T} K_{1} \\
& \alpha_{21}=-\tau \Lambda_{1} C^{T} C \Lambda_{1} \\
& \beta_{2}=M_{2}^{-1}
\end{aligned}
$$

Rewriting the second pair of equations gives

$$
\left[\begin{array}{cc}
\alpha_{11} \otimes I_{n s} & \alpha_{12} \otimes I_{n s} \\
\alpha_{21} \otimes \beta_{2} & \alpha_{22} \otimes I_{n s}
\end{array}\right]\left[\begin{array}{c}
\operatorname{col}\left(\dot{K}_{3}\right) \\
\operatorname{col}\left(\dot{\Lambda}_{3}\right)
\end{array}\right]=\left[\begin{array}{l}
0 \\
0
\end{array}\right]
$$

where the $\operatorname{col}\left(K_{3}\right)$ operation enters the elements of $K_{3}$ into a column proceeding row by row. For all $\sigma$ until a $\bar{\sigma}$ is reached at which the matrix of coefficients is singular, the only solution to these equations is $K_{3}=0, \Lambda_{3}=0$ and $K_{2}=0, \Lambda_{2}=0$. Therefore, the solution remains a direct output feedback solution until reaching $\bar{\sigma}$.

It would be of interest to know whether all of the optimal controllers of all possible dimensions $n_{c}$ are generated from the given initial conditions at $\sigma \rightarrow-\infty$ by the process of the (repeated) bifurcations described above or whether other positive (semi) definite solutions can perhaps appear spontaneously at some point or can come in from infinity, perhaps entering the positive (semi) definite region at some $\sigma$ and developing into valid solutions. It would also be of interest to understand how the solutions can disappear. For example, do solutions have to disappear by leaving the positive definite region? Some of these questions we can answer and the examples in the next section shed some light on the others.

Consider the question of whether solutions of Eqs. (6-9) associated with stabilizing controllers, so that $K \geq 0$ and $\Lambda \geq 0$, can leave the region of positive (semi) definiteness and therefore disappear as valid solutions. First, treat direct output feedback controllers. If $K$ (or $\Lambda$ ) is on the boundary of the positive definite region, then there is a zero eigenvalue with an associated eigenvector $x$ satisfying $K x=0 \quad(\Lambda x=0)$. Forming a quadratic form with this $x$ from Eqs. (25) gives

$$
\begin{aligned}
& x^{T} t \mathscr{F}(K, \Lambda,-1 / t) x=t\left(x^{T} Q x\right)=0 \\
& x^{T} t \mathcal{G}(K, \Lambda,-1 / t) x=t\left(x^{T} X x\right)=0
\end{aligned}
$$


which forms a contradiction since $Q>0$ and $X=X_{s}>0$ for $n_{c}=0$. Therefore, no solution can leave the positive definite region for $n_{c}=0$.

For $n_{c} \neq 0$, consider $K>0, \Lambda>0$ valid for some $t$. For problem $\mathbf{P}$ with $Q=$ block diag $\left[Q_{s}, O_{n c}\right], X=$ block $\operatorname{diag}\left[X_{s}, O_{n c}\right]$, and $Q_{s}>0, X_{s}>0$, Eqs. (30) imply that eigenvector $x$ satisfying $K x=0$ must have the form $x^{T}=$ $\left[0^{T}, x_{c}^{T}\right]$. The rate of change of the eigenvalue $\lambda$ is given by $x^{T}(t) \dot{K}(t) x(t)=\dot{\lambda}(t)$, assuming $x$ is normalized. Let $N(t)$ be the matrix of eigenvectors of $K(t)$ with $N^{-1}=N^{T}$ and let it be arranged so that the last column of $N$ is $x$. Taking the product of Eqs. (26) by $N^{T}$ from the left and $N$ from the right and looking at the $(n, n)$ element after freely inserting $N N^{T}$ as needed gives

$$
\left\{t x^{T}\left[(A-\bar{B} K \Lambda \bar{C})^{T}+(A-\bar{B} K \Lambda \bar{C})\right] x-2\right\} \dot{\lambda}=0
$$

This indicates that, if there is an eigenvalue $\lambda$ reaching zero, $\dot{\lambda}=0$ and, hence, stays zero, never allowing $K$ to leave the region of positive semidefiniteness-provided $\lambda$ does not become zero at a point where the coefficient above happens to be simultaneously zero. With this exception, we conclude that positive definite solutions never leave the region of positive (semi) definiteness as $t$ progresses. Furthermore, the arguments hold with $t$ running backward, from which we conclude that valid stabilizing controller solutions cannot appear at some $t$ by crossing into the positive definite region, unless the coefficient in Eq. (31) is zero when $\lambda$ is zero.

\section{Examples}

This section is devoted to solving Eqs. (6-9) for $K$ and $\Lambda$ as a function of $\sigma$ for several examples. We do not limit our search to positive definite solutions and track all solutions as $\sigma$ changes in order to get an understanding of the range of phenomena occurring. Solutions that are not positive definite are tracked with the idea that any unusual behavior they exhibit might be revealed by the positive definite solutions of interest in some other problem.

In order to simplify the search for solutions to Eqs. (6-9), system matrices are chosen that have the properties that $A^{T}=A, \bar{B}=\bar{C}$, and $Q=X$. This makes Eq. (8) obtainable from Eq. (7) by interchanging the roles of $K$ and $\Lambda$. We will make this assumption, in which case only one nonlinear matrix equation (7) needs to be solved.

The range of problem treated is somewhat more general than it might first appear. In each case, an $A_{s}$ is given and the behavior studied as $\sigma$ changes an $A+\sigma I$, including both negative and positive $\sigma$. If the $\sigma$ is changed to $\sigma=\sigma^{*}+\Delta \sigma$, then $A+\sigma I$ becomes $\left(A+\sigma^{*} I\right)+\Delta \sigma I$. For direct output feedback controllers, $\bar{A}=(A+\sigma I)$ represents a valid system matrix as well and, hence, the results obtained here apply as well to a problem with $\bar{A}$ as the system matrix by shifting the zero point of the $\sigma$ axis by $\sigma^{*}$ units.

\section{Example 1}

1) Consider first the optimization with respect to the cost function of problem $\mathrm{P}$ with $M=I, Q_{s}=1$, and $X_{s}=1$ for the first-order system

$$
\begin{gathered}
\dot{x}_{s}=a_{s} x+u \\
y=x
\end{gathered}
$$

when controlled either by a direct output feedback controller $u=h y$ or a first-order controller

$$
\begin{aligned}
& \dot{x}_{c}=a_{c} x_{c}+b_{c} y \\
& u=c_{c} x_{c}+d_{c} y
\end{aligned} \quad F=\left[\begin{array}{ll}
d_{c} & c_{c} \\
b_{c} & a_{c}
\end{array}\right]
$$

Starting with the zero-order controller, Eqs. (6-9) give

$$
\begin{aligned}
& 2\left[\left(a_{s}+\sigma\right)-k_{11} \lambda_{11}\right] k_{11}+1=0 \\
& 2\left[\left(a_{s}+\sigma\right)-k_{11} \lambda_{11}\right] \lambda_{11}+1=0
\end{aligned}
$$

Taking the difference of these equations shows that either $\left(a_{s}+\sigma\right)-k_{11} \lambda_{11}=0$, which results in the contradiction $1=0$ when substituted above, or $k_{11}=\lambda_{11}$ (so that in this case we did not have to assume $K=\Lambda$ ). Then,

$$
k_{11}^{3}-\left(a_{s}+\sigma\right) k_{11}-1 / 2=0 \text { or } k_{11}=1 / 2 /\left[k_{11}^{2}-\left(a_{s}+\sigma\right)\right]
$$

This equation appears repeatedly. The nature of its solutions can be understood by examining the second form of the equation and finding intersections of the graphs of the rightand left-hand sides as in Fig. 1. For $\left(a_{s}+\sigma\right)<0$, there is always one real solution and it is positive. When $\left(a_{s}+\sigma\right)>0$, which will happen for $\sigma \leq 0$ when the uncontrolled system is unstable, the one positive solution remains and there can be two additional solutions that are negative and hence do not correspond to solutions of the original optimization problem.

Now consider a first-order controller. Under the assumption that $K=\Lambda$, Eqs. (6-9) produce

$$
k_{11}^{3}+\left[2 k_{12}^{2}-\left(a_{s}+\sigma\right)\right] k_{11}+\left[k_{22} k_{12}^{2}-1 / 2\right]=0
$$

$$
\left[\left(a_{s}+2 \sigma\right)-2\left(k_{11}^{2}+k_{11} k_{22}+k_{22}^{2}+k_{22}^{2}+k_{12}^{2}\right)\right] k_{12}=0
$$

$$
k_{22}^{3}+\left[2 k_{12}^{2}-\sigma\right] k_{22}+\left[k_{11} k_{12}^{2}\right]=0
$$

We now attempt to find all solutions to these equations. From Eq. (34), either $k_{12}=0$ or the term in square brackets is zero. The $k_{12}=0$ choice reduces Eq. (33) to Eq. (32), which we have already solved. It reduces Eq. (35) to $\left(k_{22}^{2}-\sigma\right) k_{22}=0$ from which either $k_{22}=0$ or $k_{22}= \pm \sqrt{\sigma}$. Hence, we have three solutions

$$
\begin{array}{ll}
K=\Lambda=\left[\begin{array}{rr}
k_{11} & 0 \\
0 & 0
\end{array}\right] ; & F=-\left[\begin{array}{cc}
k_{11}^{2} & 0 \\
0 & 0
\end{array}\right] \\
K=\Lambda=\left[\begin{array}{rr}
k_{11} & 0 \\
0 & \sqrt{\sigma}
\end{array}\right] ; & F=-\left[\begin{array}{cc}
k_{11}^{2} & 0 \\
0 & \sigma
\end{array}\right] \sigma \geq 0 \\
K=A=\left[\begin{array}{rr}
k_{11} & 0 \\
0 & -\sqrt{\sigma}
\end{array}\right] ; & F=-\left[\begin{array}{cc}
k_{11}^{2} & 0 \\
0 & \sigma
\end{array}\right] \sigma \geq 0
\end{array}
$$

A bifurcation occurs at $\sigma \Rightarrow 0$ and one of the new solutions is positive definite, but all three are direct output feedback solutions in the sense that the controller state in the second two solutions is unobservable in the control signal.

Taking the second choice, making the square bracket in Eq. (34) zero, and substituting the resulting $k_{12}$ into Eqs. (33) and (35) yields after some manipulation,

$$
\begin{gathered}
\left(k_{11}+k_{22}\right)\left[\left(k_{11}+k_{22}\right)^{2}-\sigma\right]-a_{s}\left(k_{22}+1 / 2 k_{11}\right)=0 \\
\left(k_{11}+k_{22}\right)\left[\left(k_{11}+k_{22}\right)^{2}-\sigma\right]-1 / 2\left(a_{s} k_{22}-1\right)=0
\end{gathered}
$$

Obviously, if $a_{s}=0$, the equations are inconsistent and there is no solution. For $a_{s} \neq 0$, equate the second terms of these equations to find that $k_{11}+k_{22}=-1 / a_{s}$, from which we conclude that to obtain a positive definite solution $a_{s}$ must be negative. Using this sum in the equations makes two linear 
equations for $k_{11}, k_{12}$ whose solution is

$$
\begin{aligned}
& k_{11}=-\frac{2}{a_{s}^{2}}\left(\sigma-\frac{1}{a_{s}^{2}}\right)-\frac{2}{a_{s}} ; \quad k_{22}=\frac{2}{a_{s}^{2}}\left(\sigma-\frac{1}{a_{s}^{2}}\right)+\frac{1}{a_{s}} \\
& k_{12}^{2}=-\frac{4}{a_{s}^{4}}\left(\sigma-\frac{1}{a_{s}^{2}}\right)^{2}-\frac{6}{a_{s}^{3}}\left(\sigma-\frac{1}{a_{s}^{2}}\right)-\frac{3}{a_{s}^{2}}+\frac{a_{s}}{2}+\sigma
\end{aligned}
$$

These equations define two more solutions for $K, \Lambda$, and $F$ (considering both roots for $k_{12}$ ) that have a first-order controller state, provided the right-hand side of Eq. (40) is positive. One expects that this solution must have bifurcated from one of the previous solutions. Setting $k_{22}=0$ in Eq. (39) and substituting the resulting $\sigma$ into $k_{12}$ in Eq. (40) makes $k_{12}=0$, so the bifurcation occurs at $\sigma=-a_{s} / 2+1 / a_{s}^{2}$. However, if the bifurcation is to occur in the range of meaning for our problem, $\sigma<0$, then $a_{s}$ must be greater than $(2)^{1 / 3}$, which means that $k_{11}+k_{22}<0$ and the resulting solution is not positive definite.

In summary, in this example of finding and tracking all solutions of the necessary conditions [Eqs. (6-9)], we have seen that for $\sigma$ sufficiently negative the only solution is the direct output feedback solution with a gain $k_{11}^{2}$ satisfying Eq. (32) that can be seen to go to zero in the limit as $\sigma \rightarrow-\infty$, as predicted. As $\sigma$ is increased, two bifurcation points are encountered, at $\sigma=0$ and $\sigma=-a_{s} / 2+1 / a_{s}^{2}$ from which we can get a total of five solutions. One is the direct output feedback solution; two have first-order controller differential equations (the same equation in each case, one produced by a $K>0$, the other by a $K \ngtr 0$ ), but the controller state has no influence on the control signal; and two solutions are firstorder controller designs that fail to satisfy $K>0, \Lambda>0$ when the solutions exist in the $\sigma<0$ range of interest.

2) Reconsider this same example but with the cost function so that $Q=I$. We seek all solutions to the first-order controller equations. Equations (33-35) again apply, except that a $-1 / 2$ term must be inserted in Eq. (35) analogous to that in Eq. (33). As before, Eq. (34) gives us two choices for the value of $k_{12}$. Considering $k_{12}=0$ first, we see a fundamental difference between cases 1 and 2: that $k_{22}$ can no longer be zero because of the $-1 / 2$ term introduced in Eq. (35). This is reasonable-the controller state appears in the cost functional and the optimization must find a first-order controller even if it has no effect on the control signal ap-

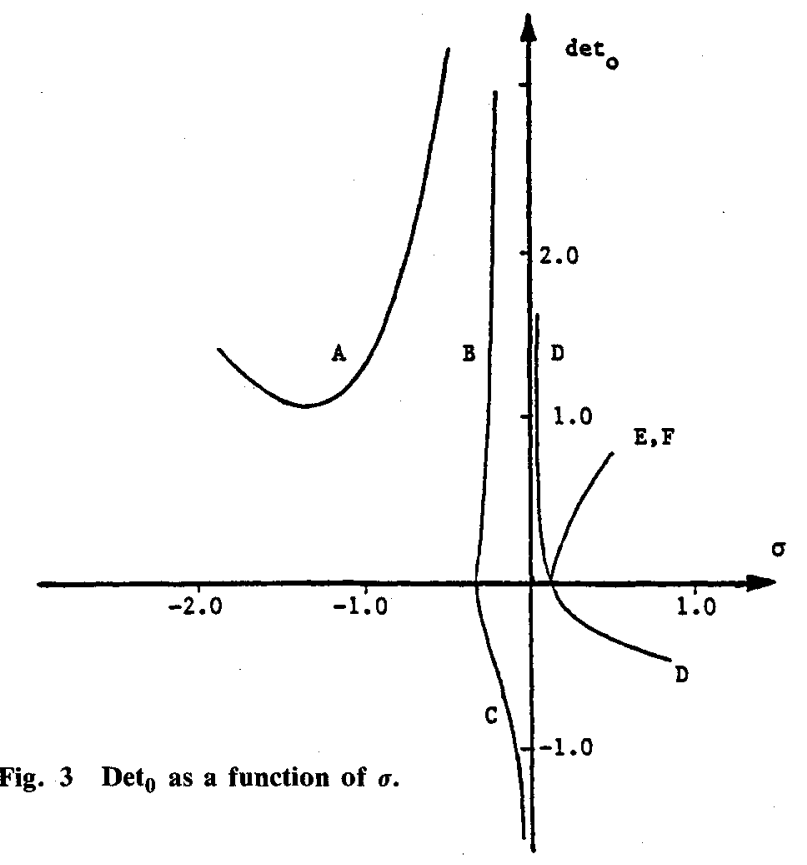

plied to $x$. These $k_{12}=0$ controllers are

$$
F=-\operatorname{diag}\left(k_{11}^{2}, k_{22}\right)
$$

where $k_{11}$ satisfies Eq. (32) and $k_{22}$ satisfies Eq. (32) with $a_{s}$ removed. For $\sigma<\min \left(-a_{s}, 0\right)$, there exists only one solution for each and each is positive. Between $-a_{s}$ and 0 , there can exist three solutions for one of the $k_{i i}$, but only one for the other, and the new solutions have negative $k_{i i}$. For $\sigma>\max \left(-a_{s}, 0\right)$ there can be three solutions for each (see Fig. 1). In all cases, the positive definite solutions give the same control signal as the direct output feedback controller obtained previously.

Other controllers are generated by the other choice of $k_{12}$ to make the square bracket in Eq. (34) zero. To find these solutions, equate term as before from Eqs. (37) and (38), but this time Eq. (37) has a $+1 / 2$ added to its left-hand side. This result is $a_{s}\left(k_{11}+k_{22}\right)=0$. Provided $a_{s} \neq 0, k_{11}=-k_{22}$. Using this to get the equation for $k_{12}^{2}$ in terms of $k_{11}$ and then eliminating $k_{12}$ and $k_{22}$ from Eq. (33) yields $k_{11}=-1 / a_{s}$. Hence,

$$
k_{11}=-1 / a_{s} ; \quad k_{22}=1 / a_{s} ; \quad k_{12}= \pm\left(\sigma+\frac{a_{s}}{2}-\frac{1}{a_{s}^{2}}\right)^{1 / 2}
$$

The square root is real if $\sigma>-a_{s} / 2+1 / a_{s}^{2}$, so these solutions bifurcate from the previous solution at the same $\sigma$ for which there was a bifurcation in case 1 of this example (the previous $\sigma=0$ bifurcation does not apply here because the pure direct output feedback controller is excluded). In fact, at the bifurxation, value of $\sigma$, the $k_{11}$ of Eq. (39) equals the $k_{11}$ in Eq. (42), as it should.

In summary, for large negative $\sigma$, there is only one solution of the form of Eq. (41) with $k_{11}$ and $k_{22}$ being positive solutions obeying the form of Fig. 1a. As $\sigma$ increases, either $k_{11}$ or $k_{22}$ can introduce a new value (as in Fig. 1b) that is not connected with the solution as $\sigma \rightarrow-\infty$. The same will happen later to the other $k_{i i}$. We conclude, in answer to one of the questions posed in the previous section, that not all solutions to Eqs. (6-9) are obtainable by bifurcations of the $\sigma$ homotopy starting at the unique solution for $\sigma \rightarrow-\infty$. Since, if $k_{11}>0, k_{22}<0$ and vice versa in Eq. (42), we see that Eq. (42) bifurcates from the positive branch of one of the $k_{i i}$ (which is obtained in the homotopy), but the other $k_{i i}$ bifurcates from the unconnected solution that has appeared. At each of the bifurcations observed, the old solution continues and two new solutions are formed.

Fig. 4 Feedback gain vs $\sigma$ for all solutions.

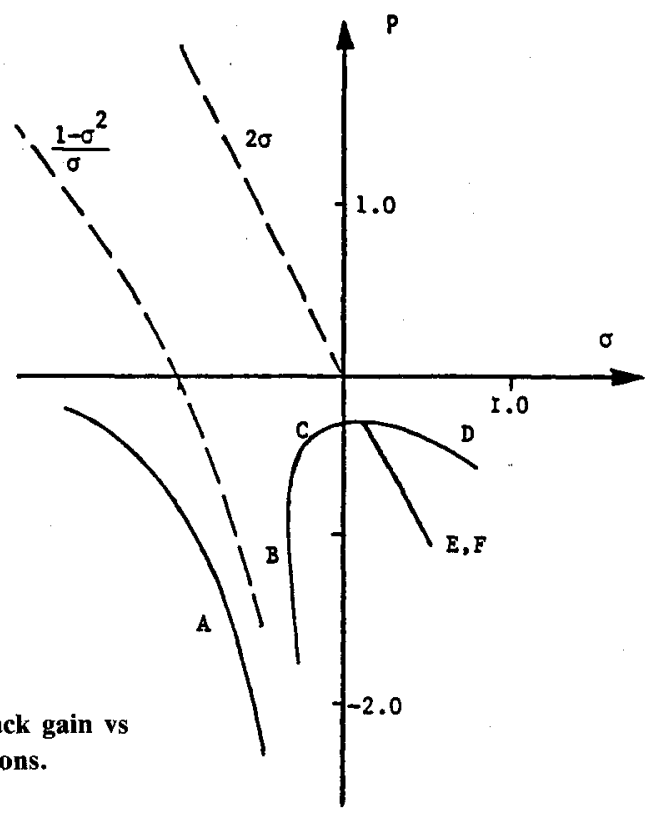




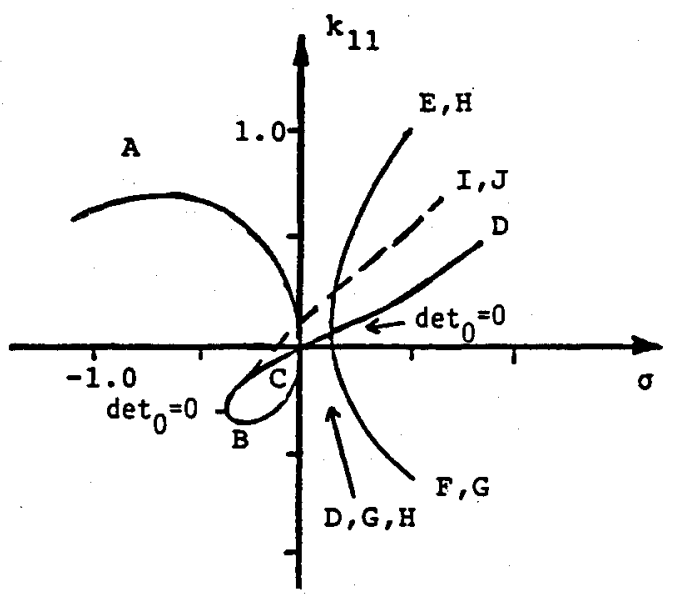

Fig. $5 \quad k_{11}$ as a function of $\sigma$ for all solutions.

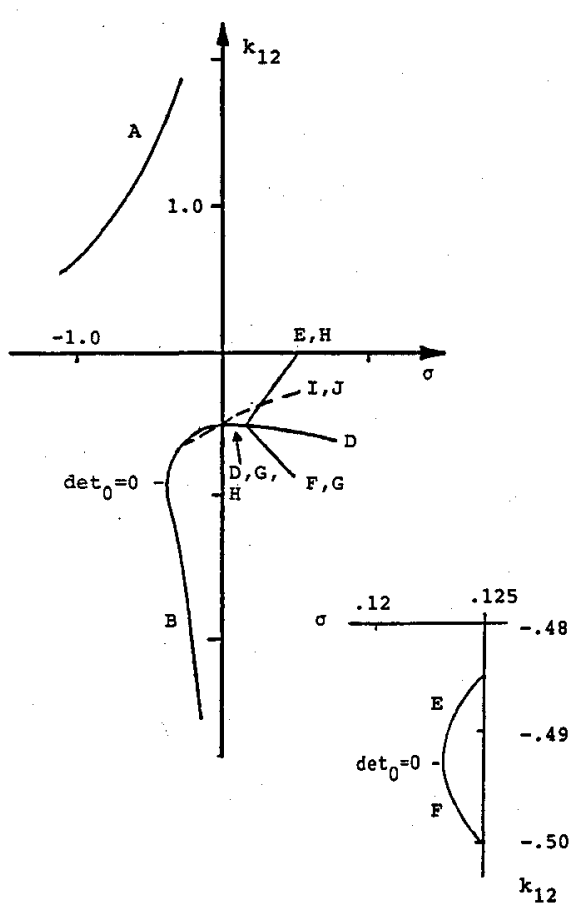

Fig. $6 k_{12}$ as a function of $\sigma$ for all solutions (detail shows bifurcation point of enlargement of solutions $E, F)$.

Still another type of pathology can be exhibited in this problem. The results above applied to $a_{s} \neq 0$. When $a_{s}=0$, Eqs. (37) (with the $1 / 2$ added) and (38), which represent Eqs. (35) and (33) with $k_{12}^{2}$ substituted, become identical. Then, in place of Eq. (42), the three unknowns $k_{11}, k_{12}$, and $k_{22}$ are any solutions of the following two equations:

$$
\begin{gathered}
\left(k_{11}+k_{22}\right)\left[\left(k_{11}+k_{22}\right)^{2}-\sigma\right]+1 / 2=0 \\
k_{12}^{2}=-\left(k_{11}+k_{22}\right)^{2}+k_{11} k_{2}+\sigma=k_{11} k_{22}+(0.5) /\left(k_{11}+k_{22}\right)
\end{gathered}
$$

Therefore, for this value of $a_{s}$ there can be an infinite number of solutions! (The second form of $k_{12}^{2}$ comes from substituting for the square term using the first equation; in the form, it is clear that we can generate solutions with $k_{12}^{2}>0$.)

If the solutions were being tracked using a differential equation such as Eq. (22), it is of interest to see the form of the equation at the bifurcation point forming Eq. (42). Substituting $k_{11}=-1 / a_{s}, \quad k_{22}=1 / a_{s}, \quad k_{12}=0$, and $\sigma=-a_{s} / 2$

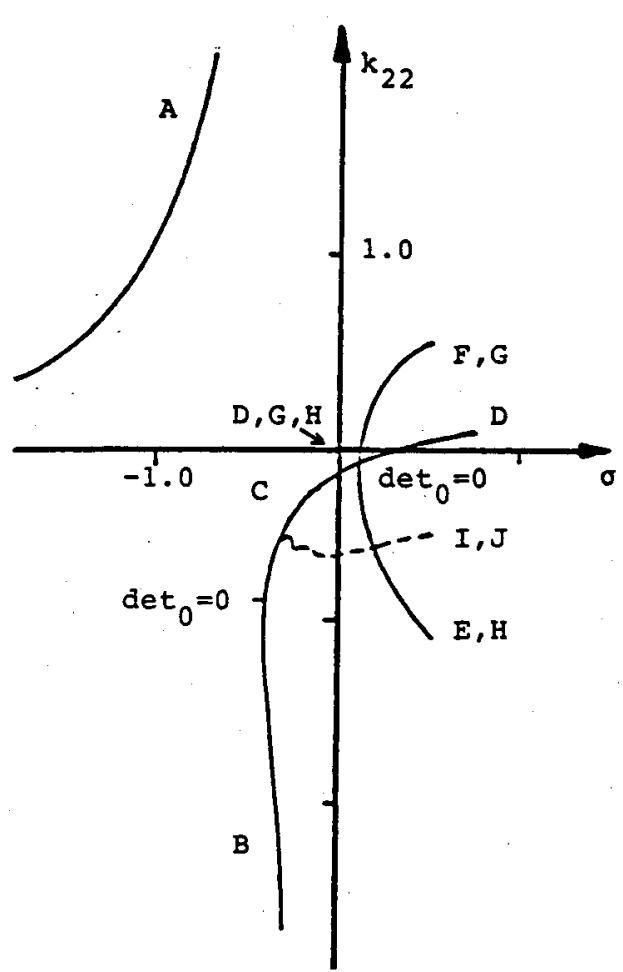

Fig. $7 \quad k_{2}$ as a function of $\sigma$ for all solutions.
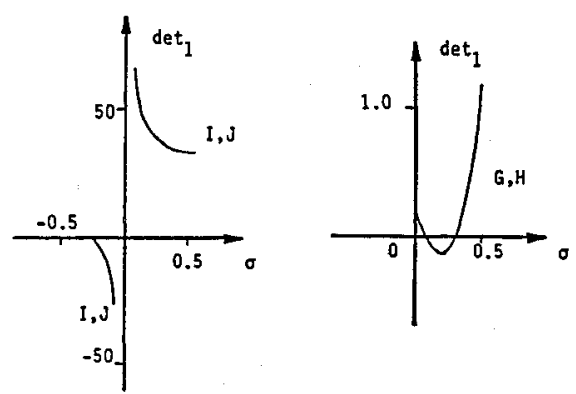

Fig. 8 First-order controller determinant det $_{1}$ as a function of $\sigma$ (enlargement shows that when det $_{1}$ reaches zero in the $\mathbf{I}, \mathbf{J}$ solution and disappears, it approaches the $\sigma$ axis vertically).

$+1 / a^{2}$ into Eq. (22) with $\beta=0$ and $K=\Lambda$ yields

$$
\left(\frac{4-a_{s}^{3}}{2 a_{s}^{2}}\right) \stackrel{\circ}{k}_{11}=\frac{1}{a_{s}} ; \quad\left(\frac{4+a_{s}^{3}}{2 a_{s}}\right) \dot{k}_{22}=\frac{1}{a_{s}} ; \quad 0 . \stackrel{\circ}{12}_{12}=0
$$

and hence $k_{12}$ can be discontinuous at this $\sigma$.

\section{Example 2}

Consider now a second-order system with system matrices and cost function matrices for problem $\mathrm{P}$ given by

$$
\begin{gathered}
A_{s}=\left[\begin{array}{ll}
0 & 1 \\
1 & 0
\end{array}\right], \quad B_{s}=\left[\begin{array}{l}
1 \\
0
\end{array}\right], \quad C_{s}=\left[\begin{array}{ll}
1 & 0
\end{array}\right] \\
X_{s}=I, \quad M=I, \quad Q_{s}=I
\end{gathered}
$$

In the case of a direct output feedback controller (with $K=\Lambda$ assumed), Eqs. (6-9) imply that

$$
\begin{gathered}
2 \sigma k_{11}+2 k_{12}+1-2 k_{11}\left(k_{11}^{2}+k_{12}^{2}\right)=0 \\
2 \sigma k_{12}+k_{22}+k_{11}-k_{12}\left(k_{11}^{2}+k_{12}^{2}\right)=0 \\
2 \sigma k_{22}+2 k_{12}+1=0
\end{gathered}
$$


Fig. 9 Values of $k_{13}, k_{23}, k_{33}$ as a function of $\sigma$ for the first-order controller solutions.
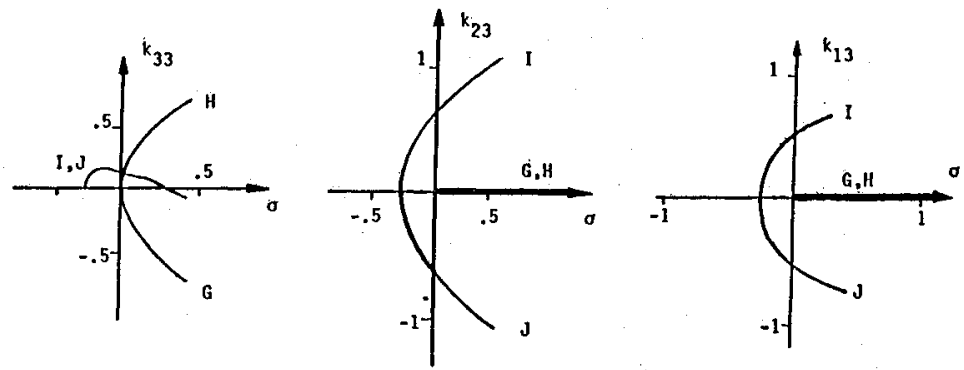

Note that, when $\sigma=0$, the system of Eq. (43) cannot be stabilized by a direct output feedback. The same is true for $\sigma>0$, but when $\sigma<0$ a feedback gain $F<\min$ $\left(-2 \sigma,\left(1-\sigma^{2}\right) / \sigma\right)$ produces stability and all positive definite solutions of Eqs. (44) must give such an $F$.

Equations (44) were solved by a root-finding algorithm and to insure that all solutions had been located, the following procedure was used. Equation (44a) is quadratic in $k_{12}$ as a function of $k_{11}$, whereas Eq. (44b) after eliminating $k_{22}$ is quadratic in $k_{11}$ as a function of $k_{12}$. For representative values of $\sigma$, these functions were plotted and the number of intersections established the total number of solutions (Fig. 2).

With $k_{22}$ eliminated, Eqs. (44a) and (44b) produce the following differential equations satisfied along the solution curves:

$$
\begin{aligned}
& {\left[\begin{array}{cc}
2 \sigma-6 k_{11}^{2}-2 k_{12}^{2} & 2-4 k_{11} k_{12} \\
1-2 k_{11} k_{12} & k_{11}^{2}+3 k_{12}^{2}+\frac{1}{\sigma}-2 \sigma
\end{array}\right]\left[\begin{array}{c}
\mathrm{d} k_{11} / \mathrm{d} \sigma \\
\mathrm{d} k_{12} / \mathrm{d} \sigma
\end{array}\right]} \\
& \quad=\left[\begin{array}{c}
-2 k_{11} \\
-\left(2+\frac{1}{\sigma^{2}}\right) k_{12}-\frac{1}{2 \sigma^{2}}
\end{array}\right]
\end{aligned}
$$

Note that solving for $k_{22}$ and substituting has introduced terms in $1 / \sigma$ which would not be present in Eq. (22) if $\mathrm{d} k_{22} / \mathrm{d} \sigma$ were also included and that this causes the determi-

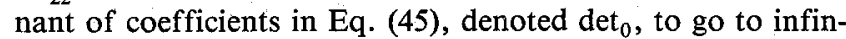
ity at $\sigma=0$.

Figure 3 gives the determinant of coefficients in Eq. (45), $\operatorname{det}_{0}$ as a function of $\sigma$ for all solutions, which are denoted A-F. The corresponding feedback gains $F$, a scalar, are given in Fig. 4. Note that the feedback gains can be well behaved at $\sigma=0$ (as can the elements of $K=\Lambda$ ), but the $\operatorname{det}_{0}$ is not well behaved for any solution. The limits on the values of $F$ for a stabilizing controller found above are also indicated and only solution $\mathrm{A}$ is stabilizing.

The corresponding solutions for $k_{11}, k_{12}$, and $k_{22}$ are given in Figs. 5-7. From Fig. 3, it is seen that the determinant of coefficients is zero at $\sigma=-0.345$ and 0.123 . These points are indicated in Figs. 5-7. The $\sigma=-0.345$ point corresponds to the spontaneous generation of a new solution unrelated to a $\sigma$ homotopy that started for large negative $\sigma$, but the new solutions generated are not positive definite. The $\sigma=+0.123$ is a point of bifurcation where the original solution continues and two new solutions start. The new solutions at the bifurcation point and the spontaneously generated new solutions all have infinite slopes $\mathrm{d} k_{11} / \mathrm{d} \sigma, \mathrm{d} k_{22} / \mathrm{d} \sigma$, and $\mathrm{d} k_{12} / \mathrm{d} \sigma$ (see detail in Fig. 6).

The equations analogous to Eqs. (44) for first-order controllers were generated and the determinant of the coefficient matrix $\left(\mathrm{det}_{1}\right)$ of the corresponding differential equation (again with $k_{22}$ eliminated as before) was monitored for first-order controllers as shown in Fig. 8. Note that the det $_{1}$ for solutions $\mathrm{I}$ and $\mathbf{J}$ depart from the $\sigma$ axis with a vertical slope which can be seen only if the scale of the figure is expanded. Solutions
$\mathrm{G}-\mathrm{I}$ were found, and the associated $k_{11}, k_{12}$, and $k_{22}$ are indicated in Figs. 5-7 and the new $k_{13}, k_{23}$, and $k_{33}$ in Fig. 9. Of the four, two have controller dynamics that are unobservable in the controller output ( $\mathrm{G}$ and $\mathrm{H}$ ) and bifurcate when $\sigma=0$, as happened in the first example. The more meaningful solutions I and $\mathrm{J}$ bifurcate from a point $\sigma=-0.26$, which is numerically near a $\operatorname{det}_{0}=0$ point and perhaps there is ill-conditioning creating the apparent difference. Five second-order controller solutions were found by the same methods to emanate from this same point (but no second-order solutions emanated from the points where the $\mathrm{G}$ and $\mathrm{H}$ solutions had det $_{1}$ zero).

\section{Conclusions}

A homotopy method was generated for obtaining stabilizing controllers of fixed dimension for linear systems. Appropriate methods for efficient performance of the homotopy were generated and a series of properties of the solutions along the homotopy were proved. Several examples were generated in which all solutions of the nonlinear matrix equations defining the stabilizing controller as a function of the homotopy parameter were found. It is interesting to note that no full-order controllers were found to be optimal in the examples (in the sense of the cost function of problem $P$ ) in spite of the fact that most modern control design methods would use a full-order controller. In these examples, the stabilizing solutions of interest were found to be connected by the homotopy to the known solutions as the homotopy parameter becomes infinitely negative. Other solutions that were not connected were found to appear suddenly when the parameter reaches certain values, but at least in these examples these unconnected solutions were not stabilizing and therefore not solutions one would be looking for. The homotopy method discussed here can be a useful technique in obtaining stabilizing and optimal fixed-order controllers, but there is a potential difficulty of having to cross bifurcation points. To minimize these difficulties, the homotopy interval should be kept short. The programming required to perform the homotopy efficiently has the extra benefit that it easily allows one to generate a Newton iteration to search for optimal controller solutions, which offers a way to speed up the rate of convergence when the gradient method is nearing a solution to the fixed-order controller problem.

\section{Acknowledgment}

Some of the work presented here was performed while the second author was with Lockheed Missiles and Space Company, Sunnyvale, CA. Their support is gratefully acknowledged.

\section{References}

${ }^{1}$ Kabamba, P. T. and Longman, R. W., "An Integrated Approach to Reduced-Order Control Theory," Optimal Control Applications and Methods, Vol. 4, 1983, pp. 405-415. (A somewhat different version appears under the same title in Proceedings of 1981 VPS \& SU/AIAA Symposium on Control of Large Flexible Spacecraft, Blacksburg, VA.)

${ }^{2}$ Ly, U.-L., Bryson, A., and Cannon, R. H., "Design of LowOrder Compensators Using Parameter Optimization," Automatica, Vol. 21, No. 3, 1985, pp. 315-318. 
${ }^{3}$ Martin, G. D. and Bryson, A. E., "Attitude Control of a Flexible Spacecraft," Journal of Guidance and Control, Vol. 3, Jan.Feb. 1980, pp. 378-441.

${ }^{4}$ Wenk, C. J. and Knapp, C. H., "Parameter Optimization in Linear Systems with Arbitrarily Constrained Controller Structure," Proceedings of IEEE Conference on Decision and Control, Dec. 1979, pp. 568-573.

${ }^{5}$ Kwakernaak, H. and Sivan, R., Linear Optimal Control Systems, Wiley, New York, 1972.

${ }^{6}$ Levine; W. S., Johnson, T. L., and Athans, M., "Optimal Limited State Variable Feedback Controllers for Linear Systems," IEEE Transactions on Automatic Control, Vol. AC-16, Dec. 1971, pp. 785-793.

${ }^{7}$ Hyland, D. C. and Bernstein, D. S., "Explicit Optimality Conditions for Fixed-Order Dynamic Compensation,"' Proceedings of 22nd IEEE Conference on Decision and Control.

${ }^{8}$ Hyland, D. C. and Bernstein, D. S., "The Optimal Projection Equations for Fixed-Order Dynamic Compensation," IEEE Transactions on Automatic Control, Vol. AC-29, 1984, pp. 1034-1037.

${ }^{9}$ Hyland, D. C. and Bernstein, D. S., "The Optimal Projection Equations for Model Reduction and the Relationships Among the Methods of Wilson, Skelton and Moore," IEEE Transactions on Automatic Control, Vol. AC-30, 1985, pp. 1201-1211.
${ }^{10}$ Bernstein, D. S., David, L. D., and Hyland, D. C., "The Optimal Projection Equation for Reduced-Order Discrete-Curve Modeling, Estimation and Control," Journal of Guidance, Control and Dynamics, Vol. 9, 1986, pp. 228-243.

${ }^{11}$ Hyland, D. C., David, L. D., Greeley, S. W., and Richter, S., "MEOP: Maximum Entropy/Optimal Projection Stochastic Modeling and Reduced-Order Design Synthesis," AFOSR-TR-F4962084-C-0015, April 1986.

${ }^{12} \mathrm{Kabamba}$, P. T. and Longman, R. W., "'Exact Pole Assignment Using Direct or Dynamic Output Feedback," IEEE Transactions on Automatic Control, V.ol. AC-27, No. 6, 1982, pp. 1244-1246.

${ }^{13}$ Longman, R. W. and Gu, Z.-Q., "Direct or Dynamic Output Feedback Pole Placement with Optimization," to be published.

${ }^{14}$ Richter, S. L. and DeCarlo; R. A., "Continuation Methods: Theory and Applications," IEEE Transactions of Circuits and Systems, Vol. CAS-30, No. 6, June 1983, pp. 347-352.

${ }^{15}$ Garcia, C. B. and Zangwill, W. I., Pathways to Solutions, Fixed Points and Equilibria, Prentice-Hall, Englewood Cliffs, NJ, 1981.

${ }^{16}$ Nering, E. D., Linear Algebra and Matrix Theory, Wiley, New York, 1963.

${ }^{17}$ Baumgarte, J., "Stabilization of Constraints and Integrals of Motion in Dynamical Systems," Computer Methods in Applied Mechanics and Engineering, Vol. 1, 1972, pp. 1-16.

\title{
From the AIAA Progress in Astronautics and Aeronautics Series...
}

\section{SATELLITE COMUNICATIONS:}

\author{
FUTURE SYSTEMS-v. 54 \\ ADVANCED TECHNOLOGIES-v. 55
}

\author{
Edited by David Jarett, TRW, Inc.
}

Volume 54 and its companion Volume 55, provide a comprehensive treatment of the satelitite communication systems that are expected to be operational in the 1980 's and of the technologies that will make these new systems possible. Cost effectiveness is emphasized in each volume, along with the technical content.

Volume 54 on future systems contains authoritative papers on future communication satellite systems in each of the following four classes: North American Domestic Systems, Intelsat Systems, National and Regional Systems, and Defense Systems. A significant part of the material has never been published before. Volume 54 also contains a comprehensive chapter on launch vehicles and facilities, from present-day expendable launch vehicles through the still developing Space Shuttle and the Intermediate Upper Stage, and on to alternative space transportation systems for geostationary payloads. All of these present options and choices for the communications satellite engineer. The last chapter in Volume 54 contains a number of papers dealing with advanced system concepts, again treating topics either not previously published or extensions. of previously published works.

Volume 55 on advanced technologies presents a series of new and relevant papers on advanced spacecraft engineering mechanics, representing advances in the state of the art. It includes new and improved spacecraft attitude control subsystems, spacecraft electrical power, propulsion subsystems, spacecraft antennas, spacecraft RF subsystems, and new earth station technologies. Other topics are the relatively unappreciated effects of high-frequency wind gusts on earth station antenna tracking performance, multiple-beam antennas for higher frequency bands, and automatic compensation of crosspolarization cuoupling in satellite communication systems.

With the exception of the first "visionary" paper in Volume 54, all of these papers were selected from the 1976 AIAA/CASI 6th Communication Satellite Systems Conference held in Montreal, Cnada, in April 1976, and were revised and updated to fit the theme of communication satellites for the 1980's. These archive volumes should form a valuable addition to a communication engineer's active library.

Volume 54, Published in 1977, 541 pp., $6 \times 9$ illus., $\$ 25.00 \mathrm{Mem} ., \$ 45.00$ List Volume 55, Published in 1977, 489 pp., $6 \times 9$ illus., $\$ 25.00$ Mem., $\$ 45.00$ List 